\title{
SALUD MENTAL EN MÉDICOS QUE REALIZAN EL SERVICIO RURAL, URBANO-MARGINAL EN SALUD EN EL PERÚ: UN ESTUDIO DE LÍNEA BASE
}

\author{
Edén Galán-Rodas 1,2,a, Juan Antonio Gálvez-Buccollini',3,4,a, Favio Vega-Galdós ${ }^{2,5, c}$, Jorge Osada ${ }^{2,6, b}$, \\ Daisy Guerrero-Padilla ${ }^{1,2, a}$, Johann Vega-Dienstmaier ${ }^{2,6,10, c}$, Lety Talledo $2,7, a$, \\ Manuel Catacora $2,8, b, c$, Fabián Fiestas $2,9, b$
}

\begin{abstract}
RESUMEN
Las condiciones poco favorables que el médico joven encara durante el Servicio Rural, Urbano Marginal en Salud (SERUMS) pueden incrementar el riesgo de enfermedades mentales. El objetivo del estudio es establecer la línea base sobre niveles de depresión y uso problemático de alcohol en aquellos médicos que realizarán dicho servicio durante el periodo 2011-2012. Se aplicó como herramientas de tamizaje la escala del Centro de Estudios Epidemiológicos para depresión y el Test para la Identificación de Trastornos por Uso de Alcohol, a 493 médicos. Se encontró que $26 \%$ de las mujeres y $14,5 \%$ de los varones puntuaron en el rango de depresión; $22 \%$ de mujeres y $26 \%$ de varones lo hicieron para uso problemático de alcohol. 39\% puntuó para cualquiera de ambas entidades. Los problemas de salud mental son comunes entre los médicos que se disponen a migrar para realizar el SERUMS, lo cual debe ser atendido para evitar mayores riesgos.
\end{abstract}

Palabras clave: Depresión; Alcoholismo; Servicios rurales de salud; Trastorno depresivo; Médicos (fuente: DeCS BIREME).

\section{MENTAL HEALTH IN PHYSICIANS DOING THE RURAL AND SUBURBAN HEALTH SERVICE IN PERU: A BASELINE STUDY}

\begin{abstract}
The disadvantageous conditions in which young physicians have to do their rural and sub-urban health service (SERUMS) may put them in a high risk for mental disorders. This study aims to establish the baseline levels of depression and alcohol use problems among those physicians scheduled to complete their SERUMS during the period 2011-2012. The Center for Epidemiologic Studies Depression Scale (CES-D) and the Alcohol Use Disorders Identification Test (AUDIT) were administered as screening tests to 493 physicians. Depression scores were met by $26 \%$ females and $14.5 \%$ males, and alcohol use problem scores were met by $22 \%$ females and $26 \%$ males. Overall, $39 \%$ persons scored for either of both mental health entities. Mental health problems seem to be common among young physicians scheduled to migrate to their SERUMS. These problems must be addressed to avoid greater risks.
\end{abstract}

Key words: Depression; Alcoholism; Rural health services; Depressive disorder; Physicians (source: MeSH NLM).

\footnotetext{
Comité del Médico Joven. Colegio Médico del Perú. Lima, Perú.

2 Red para la Acción y Avance de la Salud Mental y Psiquiatría (Red AVANSE-PSI). Lima, Perú.

Harvard South Shore Psychiatry Residency Training Program, Harvard Medical School. Boston, Massachusetts, Estados Unidos de Norteamérica.

4 Veterans Affairs Boston Healthcare System. Boston, Massachusetts, Estados Unidos de Norteamérica.

5 Departamento de Rehabilitación. Instituto Nacional de Salud Mental "Honorio Delgado - Hideyo Noguchi", Facultad de Medicina San Fernando UNMSM. Lima, Perú.

6 Facultad de Medicina Alberto Hurtado, Universidad Peruana Cayetano Heredia. Lima, Perú.

7 Puesto de Salud Alegría, Distrito las Piedras, Tambopata. Madre de Dios, Perú.

8 Oficina de Apoyo a la Docencia e Investigación, Hospital Hermilio Valdizán. Lima, Perú.

9 Laboratorios de Investigación y Desarrollo, Universidad Peruana Cayetano Heredia. Lima, Perú.

10 Hospital Nacional Cayetano Heredia. Lima, Perú.

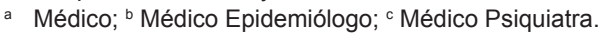

Recibido: 20-05-11 Aprobado: 15-06-11 


\section{INTRODUCCIÓN}

Los trastornos de salud mental significan aproximadamente 830000 años perdidos por discapacidad o muerte prematura, lo que coloca a estas afecciones como el grupo de enfermedades que más pérdidas económicas le significan al Perú (1). Así, los trastornos por uso de alcohol y la depresión mayor constituyen dos de las cinco primeras causas de carga de enfermedad en nuestro país. Además de ser prevalentes y estar distribuidos de manera general en la población, los trastornos mentales pueden localizarse con mayor probabilidad en grupos específicos como por ejemplo, en los migrantes ${ }^{(2)}$.

Se conoce poco acerca de la frecuencia, factores de riesgo y pronóstico de enfermedades mentales en el personal de salud ${ }^{(3)}$. Sin embargo, la exposición a niveles altos de estrés laboral hace sospechar que este tipo de profesionales podrían tener un mayor riesgo de padecer algún trastorno mental. Además, algunos reportes han encontrado que los médicos, por ejemplo, tienen serias limitaciones para acceder a servicios de salud mental cuando lo requieren ${ }^{(4)}$. Esta realidad, poco estudiada en el Perú, representa una brecha de conocimiento, especialmente para circunstancias diferentes y desventajosas para la práctica médica, como es el Servicio Rural Urbano Marginal en Salud (SERUMS).

EI SERUMS es un programa de servicio a la comunidad que está orientado a desarrollar actividades preventivopromocionales y de atención en centros y puestos de salud del Ministerio de Salud (MINSA), seguro social (EsSALUD) y de las fuerzas armadas y policiales. Este servicio se desarrolla por 12 meses, principalmente en distritos de extrema pobreza, muy pobres o pobres según la escala del mapa de pobreza del Programa Nacional Fondo Nacional de Cooperación para el Desarrollo (FONCODES). En el caso de los médicos, las plazas son adjudicadas en estricto orden de mérito considerando el promedio ponderado promocional y el examen nacional de medicina. (Decreto Supremo N. ${ }^{\circ} 007-2008-$ $\mathrm{SA}$, que aprueba modificaciones al Reglamento de la Ley N. ${ }^{\circ} 23330$, Ley del Servicio Rural y Urbano Marginal de Salud - SERUMS; ftp://ftp2.minsa.gob.pe/normaslegales/2008/DS007-2008.pdf). EI SERUMS es obligatorio para todo profesional de salud que quiera luego laborar en el sector público, así como realizar la especialización en medicina o percibir algún tipo de beca o subvención estatal (Ley N. ${ }^{\circ}$ 23330, http://www.minsa.gob.pe/servicios/serums/2011/informacion.asp). De esta manera, el SERUMS representa una situación laboral única para los médicos y otros profesionales de la salud en el Perú.

Para realizar el SERUMS, el presente año migrarán a localidades urbano-marginales o rurales lejanas el $98,5 \%$ de los 1830 médicos considerados en este programa (Datos proporcionados por la Dirección de Recursos Humanos en Salud - MINSA). El 2,5\% restante lo realizarán en distritos más urbanizados o capitales de provincias.

Las condiciones de trabajo en las localidades a las que los jóvenes médicos deben migrar para realizar el SERUMS son difíciles por la escasez de recursos logísticos y humanos para la práctica médica. Además, el desconocimiento que tienen de la geografía y los factores socioculturales en dichas localidades hace que la adaptación sea aun más difícil. Estas son condiciones para las cuales el profesional generalmente no ha sido preparado durante su entrenamiento en las escuelas de medicina. Asimismo, los "serumistas" (llamados así localmente) pueden encontrar condiciones de aislamiento y de pocas redes de soporte social, lo cual frecuentemente incluye la condición de estar incomunicados de sus seres queridos por largos periodos.

Estas condiciones hacen que el SERUMS sea considerado una circunstancia altamente estresante, además de conllevar al riesgo de una mayor incidencia de problemas de salud y muerte prematura entre los médicos que lo realizan ${ }^{(5)}$. Debido a que los niveles altos de estrés son perjudiciales para la salud mental, es razonable sospechar que el SERUMS puede estar colocando en mayor riesgo de trastornos mentales a los médicos jóvenes, que constituyen un recurso humano muy valioso y que como sociedad debemos cuidar.

\section{EL ESTUDIO}

El presente estudio tuvo como objetivo establecer una línea de base sobre niveles de síntomas depresivos y uso problemático de alcohol de todos aquellos médicos jóvenes que se disponen a realizar el SERUMS durante el periodo 2011-2012. El Decanato del Colegio Médico del Perú (CMP) aprobó que este estudio se llevara a cabo durante el VI Curso Nacional de Inducción al SERUMS en abril de 2011.

En la población de estudio están los médicos que se disponen a realizar el SERUMS durante el periodo 20112012 (censo de 1830 personas). El marco muestral (i.e., de donde se seleccionó a los participantes) fue elegido por conveniencia y estuvo constituido por aquellos médicos que se inscribieron al curso antes mencionado $(n=850)$, de los cuales se determinó a priori seleccionar a todos aquellos que asistieran el segundo día al curso. Así, se invitó a participar a los 520 presentes en el curso dicho día, de los cuales 493 aceptaron participar (proporción de rechazo: $5,2 \%$ ). 
Para el tamizaje de depresión se usó la escala del Centro de Estudios Epidemiológicos (CES-D) que consta de 20 ítems. Esta escala fue validada en Lima por Ruiz-Grosso et al. ${ }^{(6)}$, quienes encontraron que para un punto de corte (PC) de 23,5 la sensibilidad es $91,4 \%$ y la especificidad es $96,7 \%$ para detectar depresión unipolar. Para identificar uso problemático de alcohol, utilizamos el test para la identificación de trastornos por uso de alcohol de tres ítems (AUDIT-C). Gual et al. ${ }^{(7)}$, encontraron que un PC de 4,5 en el AUDIT-C para varones obtuvo una sensibilidad de $92 \%$ y una especificidad de $52 \%$ para detectar uso problemático de alcohol; mientras que un PC de 3,5 para mujeres tuvo una sensibilidad de $91 \%$ y una especificidad de $68 \%$ para detectar dicho problema. Cabe destacar que estas pruebas son herramientas de tamizaje y no de diagnóstico.

Para el análisis estadístico, las variables correspondientes a los niveles de depresión y uso problemático de alcohol fueron dicotomizadas usando los puntos de corte sugeridos. Luego, se hizo un análisis descriptivo de las variables usando porcentajes para aquellas variables dicotómicas y medidas de tendencia central y dispersión para variables continuas. La posibilidad de sesgos en este estudio, especialmente el sesgo de selección, será evaluada primariamente mediante dos puntos de referencia. El primero constituye en la comparación de las frecuencias de características demográficas entre los participantes en este estudio y las de médicos que obtuvieron su colegiatura en los últimos tres meses, ya que estos representan la población de la que derivan casi la totalidad de médicos serumistas para el periodo mayo 2011-abril 2012. El segundo punto de referencia será mediante la exploración de la validez externa de los resultados del presente estudio. Para ello, se tomó al sexo como un marcador de potenciales sesgos en el estudio, ya que su asociación con cada uno de los trastornos explorados está establecida consistentemente en la literatura, por lo que si encontráramos asociaciones diferentes a las conocidas entre estas enfermedades y el sexo, ello nos podría estar sugiriendo la presencia de sesgos que puedan estar afectando severamente la validez de los resultados de este estudio. Esta exploración se logró realizando una regresión logística multivariada para establecer si el sexo efectivamente está independientemente asociado con un mayor riesgo de presentar cada uno de los problemas de salud mental explorados, luego de ajustar el modelo por la edad y el tipo de universidad.

\section{HALLAZGOS}

De los 493 participantes en la muestra de estudio, el $55,2 \%(n=272)$ de los que respondieron fueron mujeres y la edad promedio fue de 26 años $\pm 2,6$. El $64,3 \%$ provenía de una universidad privada, el $30,2 \%$ de una pública y el 5,5\% del extranjero. El 96,8\% tuvo estado civil soltero (Tabla). Por otro lado, de los 2233 médicos que habían obtenido la colegiatura en los últimos tres meses previos al día de la encuesta, 50,4\% fueron mujeres, $63,8 \%$ provino de una universidad privada, $32,3 \%$ de

Tabla. Distribución de características demográficas por categorías de puntajes de depresión en el CES-D y de uso problemático de alcohol en el AUDIT.

\begin{tabular}{|c|c|c|c|c|}
\hline & \multicolumn{2}{|c|}{ Tamizaje para depression (CES-D) } & \multicolumn{2}{|c|}{ Tamizaje para uso problemático de alcohol (AUDIT) } \\
\hline & Negativo (390) & Positivo (103) & Negativo (377) & Positivo (116) \\
\hline Edad $(X+D E)$ & $26,0(2,7)$ & $25,9(2,6)$ & $26,2(2,8)$ & $25,5(1,8)$ \\
\hline \multicolumn{5}{|l|}{ Sexo n (\%) } \\
\hline Mujeres & $201(73,9)$ & $71(26,1)$ & $213(78,3)$ & $59(21,7)$ \\
\hline Varones & $189(85,5)$ & $32(14,5)$ & $164(74,2)$ & $57(25,8)$ \\
\hline \multicolumn{5}{|c|}{ Tipo de universidad n (\%) } \\
\hline Privada & $237(74,8)$ & $80(25,2)$ & $231(72,9)$ & $26(27,1)$ \\
\hline Estatal & $130(87,3)$ & $19(12,7)$ & $127(85,2)$ & $22(14,8)$ \\
\hline Extranjera & $23(85,2)$ & $4(14,8)$ & $19(70,4)$ & $8(29,6)$ \\
\hline \multicolumn{5}{|l|}{ Estado civil n (\%) } \\
\hline Soltero & $377(79,9)$ & $100(20,1)$ & $362(75,9)$ & $115(24,1)$ \\
\hline Casado & $3(100)$ & 0 & $3(100)$ & 0 \\
\hline Conviviente & $9(75,0)$ & $3(25,0)$ & $11(91,7)$ & $1(8,3)$ \\
\hline Otro & $1(100)$ & 0 & $1(100)$ & 0 \\
\hline
\end{tabular}


una pública y 3,9\% del extranjero (datos proporcionados por el Área de Sistema e Informática del CMP).

\section{DEPRESIÓN}

Se encontró que $26 \%$ de las mujeres y $14,5 \%$ de los varones puntuaron en el rango de depresión. Asimismo, se encontró en el análisis multivariado que, manteniendo constantes la edad y tipo universidad de procedencia (i.e., privada, pública o extranjera), el ser mujer estuvo asociado con un mayor riesgo de depresión $(\mathrm{OR}=2,0 ; \mathrm{IC} 95 \%=1,26 ; 3,25 ; \mathrm{p}=0,003)$ con respecto a los varones.

\section{ALCOHOL}

Con puntos de corte diferenciados por sexo en el AUDIT encontramos que $22 \%$ de mujeres y $26 \%$ de varones puntuaron en el rango de uso problemático de alcohol, el cual incluye uso de riesgo, uso dañino y probable dependencia al alcohol. En el análisis multivariado, luego de ajustar por edad y tipo de universidad de procedencia, se encontró que los varones tuvieron una tendencia mayor que las mujeres de tener un uso problemático de alcohol, aunque esta tendencia no alcanzó a tener significancia estadística $(O R=1,4 ; \operatorname{IC} 95 \%=0,9 ; 2,2$; $p=0,103)$.

En general, 39\% de los entrevistados puntuaron en los instrumentos de tamizaje en el rango de al menos uno de los problemas, depresión o uso problemático de alcohol, porcentaje que incluye un $5 \%$ con ambas condiciones.

\section{DISCUSIÓN}

Este estudio tuvo el objetivo de establecer la línea base de la salud mental en médicos recién graduados que se encuentran próximos a migrar a regiones rurales y alejadas del país para realizar el SERUMS. En este tamizaje encontramos que la depresión y el uso problemático de alcohol serían muy prevalentes en esta población, aun más que la que se reporta para la población general $(8,9)$. Específicamente, encontramos que aproximadamente dos de cada cinco médicos serumistas tendrían una salud mental deteriorada antes de migrar para el ejercicio de la profesión en lugares alejados de sus lugares de residencia.

Algunas limitaciones y fortalezas deben considerarse antes de discutir las implicancias de este estudio. La principal limitación es la posibilidad de un sesgo de selección debido al muestreo por conveniencia que se usó en este estudio. Hubiera sido ideal tener una muestra aleatoria de todos los que se disponen a hacer el SERUMS este año, pero ello no fue posible implementar por la limitación de los recursos con los que se contó al momento de realizarse la encuesta. Si bien es cierto que el muestreo puede haber causado que la muestra final no sea representativa de la población de estudio, hay puntos referenciales que nos hacen tener confianza en que el sesgo de selección no es lo suficientemente severo como para tener un impacto importante en la validez de los hallazgos.

Primero, es improbable que las razones de no participación estén relacionadas con el tema del estudio, pues este se presentó a la audiencia solo el mismo día de aplicación de la encuesta. Segundo, la distribución por sexo y tipo de universidad de procedencia de los participantes fueron muy similares a la que el CMP reporta para aquellos que se colegiaron el primer trimestre del año 2011. Por último, la asociación del sexo con la depresión y con el uso problemático de alcohol es consistente con la literatura ${ }^{(10,11)}$. Todo ello nos da confianza de la validez de nuestros resultados.

Sin embargo, no podemos descartar completamente que un sesgo de selección pueda estar influenciando de manera importante nuestros resultados. Si fuera este el caso, creemos que el sesgo de selección nos estaría llevando a tener una sobrerrepresentación en la muestra de personas sin problemas de salud mental, ya que las personas que no tienen problemas de salud mental tienen mayor probabilidad de participar de actividades como el curso organizado por el CMP en donde se recolectó la muestra. Ello significaría entonces que nuestra estimación de que aproximadamente $39 \%$ de médicos serumistas podrían tener depresión o un uso problemático de alcohol es subestimada y que en la realidad podría ser más prevalente.

Por otro lado, en cuanto a las fortalezas, a nuestro conocimiento el presente estudio es el primero que explora la salud mental en médicos en el Perú, especialmente aquellos que se prestan a migrar para realizar el SERUMS. Los estudios más cercanos son aquellos enfocados en estudiantes de medicina y mayormente centradas en depresión ${ }^{(12-14)} y$, en su mayoría, encuentran frecuencias de depresión tan altas como en este estudio. Por último, este reporte presenta un punto de referencia para estudios longitudinales futuros de incidencia y trayectoria de trastornos mentales una vez que estos médicos han iniciado sus actividades de SERUMS.

En conclusión, usando herramientas de tamizaje, el presente estudio encuentra altos niveles de depresión y uso problemático de alcohol para los médicos que se prestan a migrar para iniciar el SERUMS. Ello significa 
un punto de partida desventajoso ya que el SERUMS se asocia con condiciones difíciles y escasos recursos de afronte. Esto genera preocupación dado el alto riesgo que existe de que estos problemas empeoren en dicho periodo debido a las limitadas posibilidades de recibir ayuda profesional durante el desarrollo de la actividad. Nuestros hallazgos llevan a recomendar que el Ministerio de Salud y otras instituciones implementen estrategias integrales para cuidar la salud mental de este recurso humano médico vulnerable. Una recomendación puntual es permitir que los serumistas puedan recibir ayuda profesional usando el sistema de seguro de salud (ESSALUD) desde el primer mes de su inclusión y aportes en dicho sistema y no esperar hasta el tercer mes como está establecido actualmente (http://www.essalud. gob.pe/contenidoframe.php?id=3). Asimismo, las universidades deberían implementar o mejorar los programas de autocuidado y prevención de problemas de salud mental entre los estudiantes de medicina ${ }^{(15)}$. Todo ello innegablemente incidirá en mejorar no solo el bienestar de los médicos serumistas, sino también la calidad de atención en las poblaciones más olvidadas en nuestro país, que son a quienes ellos brindan su ayuda.

\section{Contribuciones de Autoría}

Todos los autores idearon y diseñaron el estudio. EGR y DGP recolectaron y construyeron la base de datos. JAGB y FF analizaron los datos. EGR, JAGB y FF escribieron la primera versión del artículo. Todos los autores revisaron críticamente la serie de versiones del artículo. Todos los autores leyeron y aprueban la versión final del artículo.

\section{Fuentes de Financiamiento}

Autofinanciado

\section{Conflictos de Interés}

Los autores declaran no tener conflictos de interés relacionado con el tema del presente artículo.

\section{REFERENCIAS BIBLIOGRÁFICAS}

1. Velásquez A. La carga de enfermedad y lesiones en el Peru y las otras prioridades del plan esencial de aseguramiento universal. Rev Peru Med Exp Salud Publica. 2009;26(2):222-31.

2. Breslau J, Borges G, Tancredi D, Saito N, Kravitz R, Hinton L, et al. Migration from Mexico to the United States and subsequent risk for depressive and anxiety disorders: a crossnational study. Arch Gen Psychiatry. 2011;68(4):428-33.

3. Miller L. Doctors, their mental health and capacity for work. Occup Med (Lond). 2009;59(1):53-5.

4. Kay M, Mitchell G, Clavarino A, Doust J. Doctors as patients: a systematic review of doctors' health access and the barriers they experience. British Journal of General Practice. 2008 Jul;58(552):501-8.

5. Galan-Rodas E, Diaz-Velez C, Villena J, Maguina C. Mortalidad de médicos que Realizan el servicio rural (SERUMS) en Perú, 2006-2009. Rev Peru Med Exp Salud Publica. 2010;27(3):483-4.

6. Ruiz-Grosso P, Vega-Dienstmaier JM, Loret de Mola C, Arévalo-Flores M, Zapata-Vega M. Validación de la escala para depresión del Centro de Estudios Epidemiológicos (CES-D) en un hospital nacional en Lima, Perú. XX Congreso Peruano de Psiquiatría. Lima: Asociación Psiquiátrica Peruana; 2008.

7. Gual A, Segura L, Contel M, Heather N, Colom J. Audit-3 and audit-4: effectiveness of two short forms of the alcohol use disorders identification test. Alcohol Alcohol. 2002;37(6):591-6.

8. Instituto Nacional de Salud Mental. Estudio epidemiológico en salud mental 2002. Anales de Salud Mental. 2002;18(1,2):13-81.

9. Galvez-Buccollini JA, Paz-Soldan VA, Herrera PM, DeLea S, Gilman RH. Gender differences in sex-related alcohol expectancies in young adults from a peri-urban area in Lima, Peru. Rev Panam Salud Publica. 2009;25(6):499-505.

10. Degenhardt L, Chiu WT, Sampson N, Kessler RC, Anthony JC, Angermeyer M, et al. Toward a global view of alcohol, tobacco, cannabis, and cocaine use: findings from the WHO World Mental Health Surveys. PLoS Med. 2008;5(7):e141.

11. Weissman MM, Bland RC, Canino GJ, Faravelli C, Greenwald S, Hwu HG, et al. Cross-national epidemiology of major depression and bipolar disorder. Jama. 1996;276(4):293-9.

12. Galli-Silva E, Feijoo-Llontop L, Roig-Rojas I, Romero-EImore S. Aplicación del "MINI" como orientación diagnóstica psiquiátrica en estudiantes de medicina de la Universidad Peruana Cayetano Heredia: informe preliminar epidemiológico. Rev Med Hered 2002;13(1):19-25.

13. Pereyra-Elias R, Ocampo-Mascaro J, Silva-Salazar V, Velez-Segovia E, Costa-Bullon AD, Toro-Polo LM, et al. Prevalencia y factores asociados con síntomas depresivos en estudiantes deficiencias de la salud de una Universidad privada de Lima, Perú 2010. Rev Peru Med Exp Salud Publica. 2010;27(4):520-6.

14. Castillo-Vilca M, Prado-Mendoza C, Vega-Dienstmaier JM. Prevalencia de depresión en estudiantes del quinto año de medicina de una universidad privada de Lima. Rev Neuropsiquiatr. 2010;73(1):9-14.

15. Voltmer E, Kieschke U, Schwappach DL, Wirsching M, Spahn C. Psychosocial health risk factors and resources of medical students and physicians: a cross-sectional study. BMC Med Educ. 2008;8:46.

Correspondencia: Fabian Fiestas

Dirección: Laboratorios de Investigación y Desarrollo,

Universidad Peruana Cayetano Heredia.

Av. Honorio Delgado 430, San Martín de Porres, Lima 31, Perú.

Teléfono: (511) 985-765743

Correo electrónico: ffiestas@epi.msu.edu 